Pacific

Journal of

Mathematics

NORMAL HOLONOMY AND WRITHING NUMBER OF POLYGONAL KNOTS

James J. Hebda and Chichen M. Tsau 


\title{
NORMAL HOLONOMY AND WRITHING NUMBER OF POLYGONAL KNOTS
}

\author{
James J. Hebda and Chichen M. Tsau
}

The normal holonomy of a polygonal knot is a geometrical invariant which is closely related to the writhing number. We show that normal holonomy fibers the space of knots over the circle and deduce that the writhing number fibers the space of knots over the real line. Consequently, two isotopic knots which have the same writhing number are isotopic through a family of knots having the same writhing number. In a similar vein, two isotopic knots having zero holonomy are isotopic through a family of such knots if and only if they have the same autoparallel linking number.

More generally, the definition of normal holonomy makes sense for immersed polygonal knots. This time normal holonomy fibers the space of immersed knots over the circle, but now there are only two isotopy classes of immersed knots of zero holonomy.

\section{Introduction.}

The writhing number of a smooth knot in Euclidean 3-space is a real number which measures the extent to which the knot coils around itself. Although named by F.B. Fuller [8], the writhing number was originally discovered by G. Călugăreanu [4] and [5], in the form of an integral which he obtained while investigating the behavior in the limit of the Gauss formula for the linking number of a pair of space curves as one of the curves is allowed to approach the other. In this way, he established the formula

$$
L K(K, U)=W R(K)+T W(K, U)
$$

where $U$ is a unit vector field along the knot $K, L K(K, U)$ is the linking number of $K$ with a nearby knot that approaches $K$ from a direction along $U, W R(K)$ is the writhing number, and $T W(K, U)$ is the twist of $U$ along $K$. Recently, the writhing number of a smooth knot has been related to the helicity of vector fields [6] and [17], and applied in the discussion of models of bacterial fibers [19].

The normal holonomy of the knot is a geometric invariant closely related to the writhing number, but more easily computed. Consider parallel translation with respect to the induced connection in the normal bundle of the 
knot. Parallel translating a vector that is perpendicular to the knot at some point, once around the knot, has the effect of rotating the vector through an angle in the normal plane. Every such vector is rotated through the same angle. This common "phase" is the normal holonomy of the knot and may be regarded variously as an angle, a real number modulo $2 \pi$, or a point on the unit circle. Suppose the knot $K$ is parametrized on the interval $[0, \ell]$. Let $X$ be a parallel unit normal vector field along $K$. The angle in the normal plane from $X_{0}$ to $X_{\ell}$ equals the normal holonomy HOL $(K)$. Suppose $U$ is a smooth unit normal vector field along $K$, and let $\theta$ be a continuous choice of angle between $X$ and $U$ along $K$. Because $U_{0}=U_{\ell}, \theta_{0}-\theta_{\ell}=\operatorname{HOL}(K)$. On the other hand, $\theta_{\ell}-\theta_{0}=2 \pi T W(K, U)$. Since $L K(K, U)$ is an integer, the formula (1.1) implies

$$
2 \pi W R(K) \equiv \operatorname{HOL}(K) \bmod 2 \pi .
$$

Thus the normal holonomy may be identified with the "fractional part" of the writhing number. The connection of writhe to the normal holonomy or Berry's phase has appeared in the literature [15]. Earlier Banchoff and White [3] and [20], observed that the fractional part of the twist depends only on the knot. A closely related formula to (1.2) was observed by F.B. Fuller [9, Equation 6.1]. (See also [1, Equation 16].)

In this paper we study the normal holonomy and writhing number of polygonal, rather than smooth, knots. For us, polygonal knots are piecewise linear embeddings of the circle into 3-space, and so come already parametrized. This differs with the usual definition of polygonal knots as the image of such embeddings in $\mathbf{R}^{3}$, but our definition simplifies the discussion of the topology on the space of knots. This is important because our point of view is to regard the normal holonomy and writhing number as functions on the space of polygonal knots. Our main result is that normal holonomy fibers the space of knots over the circle and that writhing number fibers it over the real line.

As a corollary, we prove that two isotopic polygonal knots which have the same writhing number are isotopic through a family of knots having the same writhing number. This corollary was proved for $C^{1}$-smooth knots by Miller and Benham [16] by considering individual knots and adjusting an isotopy between them. For knots of zero holonomy, the writhe is an integer which is equal to the linking number of the knot with a nearby, geometrically determined, parallel curve. We call this integer the autoparallel linking number of the knot of zero holonomy and prove that two isotopic knots having zero holonomy are isotopic through a family of such knots if and only if they have the same autoparallel linking number.

These results may be viewed as the three dimensional version of the fact that two oriented isotopic knot diagrams are regularly isotopic if and only if they have the same writhing number and rotation number. (For a special 
case, see [14, pp. 172-173].) They also bear comparison to the result that two isotopic smooth knots of non-vanishing curvature are isotopic through a family of such knots if and only if they have the same self-linking number [10] and [11]. Smooth knots of non-vanishing curvature form an open dense subset of the space of smooth knots, while the polygonal knots of zero holonomy are nowhere dense in the space of polygonal knots.

Finally we prove that holonomy fibers the space of immersed polygonal knots over the circle. Consequently, we obtain a result concerning immersed polygonal knots of zero holonomy which is analogous to Feldman's results for smoothly immersed space curves of non-vanishing curvature [7].

\section{The normal holonomy of polygonal knots.}

A polygonal knot is a piecewise linear embedding of the circle $S^{1}$ into Euclidean 3-space $\mathbf{R}^{3}$. Thus, given a polygonal knot $K: S^{1} \rightarrow \mathbf{R}^{3}$, there is a finite subset $\sigma$ of $S^{1}$ which subdivides $S^{1}$ into a finite number of intervals, such that the mapping $K$ restricted to each of these intervals is a linear affine mapping into $\mathbf{R}^{3}$ as a function of the usual angle parameter $t \bmod 2 \pi$ in $S^{1}$. The elements of $\sigma$ are denoted $t_{0}<t_{1}<\cdots<t_{n-1}<t_{n}=t_{0}$ and are to be regarded as cyclically ordered. The point $K\left(t_{i}\right)$ is the $i$-th vertex of $K$, and the restriction of $K$ to $\left[t_{i-1}, t_{i}\right]$ is the $i$-th edge. Because of cyclic ordering, the $n$-th edge is the restriction to the interval $\left[t_{n-1}, t_{0}\right]$. Let

$$
T_{i}=\frac{K\left(t_{i}\right)-K\left(t_{i-1}\right)}{\left|K\left(t_{i}\right)-K\left(t_{i-1}\right)\right|}
$$

denote the oriented unit tangent vector along the $i$-th edge. The exterior angle $\alpha_{i}$ at the $i$-th vertex is the angle between $T_{i}$ and $T_{i+1}$ with $0 \leq \alpha_{i}<\pi$. Since $K$ is an embedding, $\alpha_{i} \neq \pi$. The definition allows for the possibility that $\alpha_{i}=0$. When $\alpha_{i} \neq 0$, the unit normal vector $\xi_{i}$ at the $i$-th vertex is defined by the cross product

$$
\xi_{i}=\frac{T_{i} \times T_{i+1}}{\left|T_{i} \times T_{i+1}\right|} .
$$

When $\alpha_{i}=0$, any unit vector $\xi_{i}$ perpendicular to $T_{i+1}=T_{i}$ may be chosen and used in the following formulas.

Let $T_{i}^{\perp}$ denote the two-dimensional space of normal vectors to the $i$-th edge, oriented so that $X, Y$ is an oriented basis if and only if $T_{i}, X, Y$ is an oriented triad in $\mathbf{R}^{3}$. Rotation about the vector $\xi_{i}$ through the angle $\alpha_{i}$ is a linear isometry that carries $T_{i}^{\perp}$ onto $T_{i+1}^{\perp}$. It is the identity when $\alpha_{i}=0$. Let $R_{i}$ denote this isometry. The composition of all these rotations

$$
R_{n} \circ \cdots \circ R_{1}: T_{1}^{\perp} \rightarrow T_{1}^{\perp}
$$

is an orientation preserving linear isometry of $T_{1}^{\perp}$, hence is a rotation of $T_{1}^{\perp}$ through some angle $\mathrm{HOL}(K)$ (regarded as a number modulo $2 \pi$ ) that we 
will call the normal holonomy of the knot $K$. Clearly, the angle HOL $(K)$ does not depend upon which edge is considered to be the first. This will be obvious from the formula we derive for $\operatorname{HOL}(K)$.

A simple formula for the normal holonomy can be obtained by the following considerations. There are two canonical, oriented orthonormal ordered bases for the two-dimensional vector space $T_{i}^{\perp}$, the alpha frame $A_{i}=\left\{\xi_{i-1}, T_{i} \times \xi_{i-1}\right\}$ and the omega frame $\Omega_{i}=\left\{\xi_{i}, T_{i} \times \xi_{i}\right\}$. One can regard these frames as the linear isometries $A_{i}, \Omega_{i}: \mathbf{R}^{2} \rightarrow T_{i}^{\perp}$ which carry the standard basis to the respective bases. By construction the matrix representing $R_{i}$ relative to the omega basis of $T_{i}^{\perp}$ and the alpha basis of $T_{i+1}^{\perp}$ is the identity matrix, that is,

$$
A_{i+1}^{-1} \circ R_{i} \circ \Omega_{i}=\left[\begin{array}{ll}
1 & 0 \\
0 & 1
\end{array}\right] \text {. }
$$

The change of basis matrix $\Phi_{i}$ between the alpha and omega frames of $T_{i}^{\perp}$ is given by rotation through some angle $\phi_{i}$ as follows:

$$
\Phi_{i}=\Omega_{i}^{-1} \circ A_{i}=\left[\begin{array}{cc}
\cos \left(\phi_{i}\right) & -\sin \left(\phi_{i}\right) \\
\sin \left(\phi_{i}\right) & \cos \left(\phi_{i}\right)
\end{array}\right] .
$$

Since the columns of a change of basis matrix are the coordinates of the old basis in terms of the new, we have

$$
\xi_{i-1}=\cos \left(\phi_{i}\right) \xi_{i}+\sin \left(\phi_{i}\right) T_{i} \times \xi_{i} .
$$

Therefore

$$
\cos \left(\phi_{i}\right)=\xi_{i} \cdot \xi_{i-1}
$$

and

$$
\sin \left(\phi_{i}\right)=T_{i} \times \xi_{i} \cdot \xi_{i-1}=T_{i} \cdot \xi_{i} \times \xi_{i-1} .
$$

This pair of formulas is sufficient to determine $\phi_{i}$ modulo $2 \pi$.

Now if we express the rotation $R_{i}$ relative to the alpha bases of $T_{i}^{\perp}$ and $T_{i+1}^{\perp}$ we obtain

$$
A_{i+1}^{-1} \circ R_{i} \circ A_{i}=A_{i+1}^{-1} \circ R_{i} \circ \Omega_{i} \circ \Omega_{i}^{-1} \circ A_{i}=\Phi_{i} .
$$

Since the holonomy is the product of the $R_{i}$, we obtain the formula

$$
\operatorname{HOL}(K)=\sum_{i=1}^{n} \phi_{i} \bmod 2 \pi .
$$

Remark. When the vertices of $K$ are in general position, $\sum_{i=1}^{n} \phi_{i}$ is equal to the negative of the total torsion $T T(K)$ defined in [2], because $-\phi_{i}$ is the signed angle from $\xi_{i-1}$ to $\xi_{i}$ by (2.5). Formula (2.6) shows that HOL $(K)$ depends continuously on the vertices of $K$. In contrast, the total torsion is discontinuous at polygonal knots whose vertices are not in general position.

Example 2.1. Planar curves have zero holonomy mod $2 \pi$. 
Example 2.2. Polygonal knot diagrams can be made into knots by replacing each crossing by a small polygonal bridge as in Figure 2.1. Knots constructed in this way clearly have zero holonomy mod $2 \pi$.

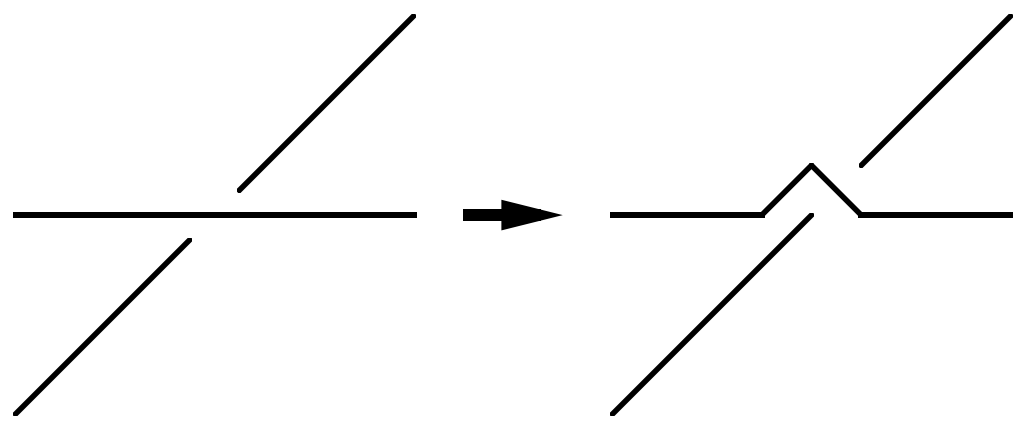

Figure 2.1

Example 2.3. Consider the 1-parameter family of curves

$$
K_{\theta}: S^{1} \rightarrow \mathbf{R}^{3}
$$

defined so that the vertices are the sequence of points $v_{0}=(0,0,0), v_{1}=$ $(0,1,0), v_{2}=(1,2,0), v_{3}=(0,3,0), v_{4}=(0,2,0), v_{5}=(-\cos \theta, 1, \sin \theta)$. Note that the first and fourth edges lie along the $y$-axis. The parameter $\theta$ is the dihedral angle between the $x y$-plane and the plane containing the triangle $v_{0} v_{4} v_{5}$. Imagine the triangle $v_{0} v_{4} v_{5}$ rotating about the $y$-axis. See Figure 2.2.

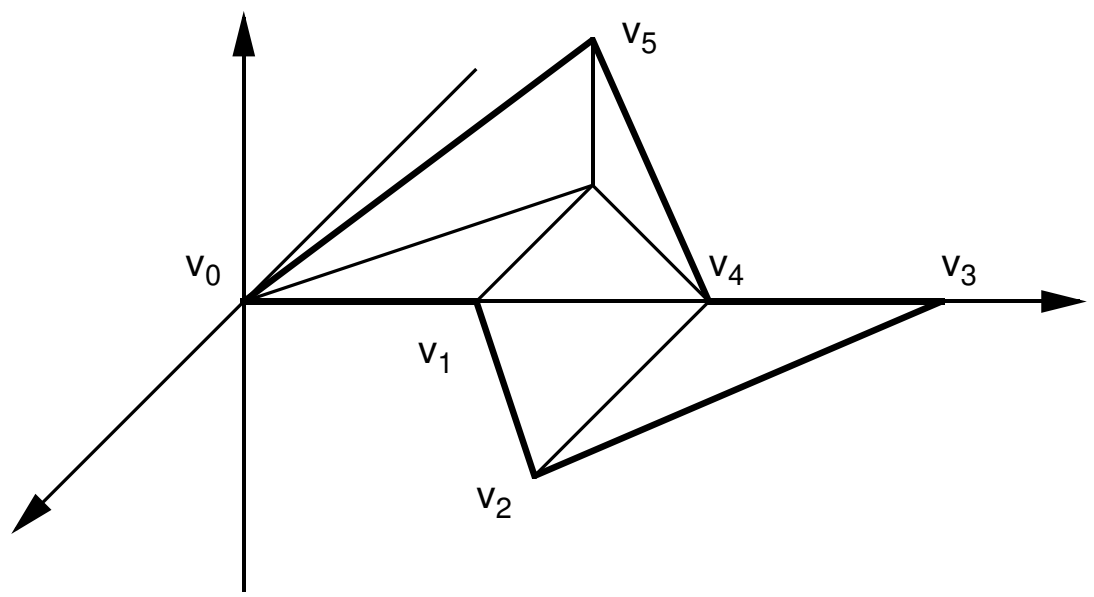

Figure 2.2 
A computation with the formulas (2.4) and (2.5) gives:

$$
\begin{aligned}
& \Phi_{1}=\Phi_{4}=\left[\begin{array}{cc}
-\cos \theta & \sin \theta \\
-\sin \theta & -\cos \theta
\end{array}\right] \\
& \Phi_{2}=\Phi_{5}=\left[\begin{array}{cc}
-1 & 0 \\
0 & -1
\end{array}\right] \\
& \Phi_{3}=\Phi_{6}=\left[\begin{array}{ll}
1 & 0 \\
0 & 1
\end{array}\right] .
\end{aligned}
$$

Thus

$$
\Phi_{6} \circ \cdots \circ \Phi_{1}=\left[\begin{array}{cc}
\cos 2 \theta & -\sin 2 \theta \\
\sin 2 \theta & \cos 2 \theta
\end{array}\right] .
$$

Therefore HOL $\left(K_{\theta}\right)=2 \theta$ modulo $2 \pi$.

Example 2.4. In this example we examine the effect on holonomy of putting a twist in an edge. Let $E_{\epsilon}^{\delta}$ be the 2-parameter family of polygonal arcs whose vertices are: $v_{0}=(0,-3,0), v_{1}=\left(0,-\frac{8 \delta}{3}, 0\right)$,

$$
\begin{gathered}
v_{2}=\left(-\frac{5 \epsilon \delta}{\sqrt{1-25 \epsilon^{2}}},-\delta, \frac{20 \epsilon \delta}{3 \sqrt{1-25 \epsilon^{2}}}\right), \quad v_{3}=\left(0,0, \frac{20 \epsilon \delta}{3 \sqrt{1-25 \epsilon^{2}}}\right), \\
v_{4}=\left(\frac{5 \epsilon \delta}{\sqrt{1-25 \epsilon^{2}}}, \delta, \frac{20 \epsilon \delta}{3 \sqrt{1-25 \epsilon^{2}}}\right), \quad v_{5}=\left(0, \frac{8 \delta}{3}, 0\right)
\end{gathered}
$$

and $v_{6}=(0,3,0)$, where $0 \leq \epsilon<\frac{1}{5}$ and $0<\delta \leq 1$. See Figure 2.3. Then the corresponding unit tangent vectors are found to be: $T_{1}=T_{6}=$ $(0,1,0), T_{2}=\left(-3 \epsilon, \sqrt{1-25 \epsilon^{2}}, 4 \epsilon\right), T_{3}=T_{4}=\left(5 \epsilon, \sqrt{1-25 \epsilon^{2}}, 0\right)$ and $T_{5}=$ $\left(-3 \epsilon, \sqrt{1-25 \epsilon^{2}},-4 \epsilon\right)$. The unit normal vectors at the 0 th, 3 rd and 6 th vertex are chosen for convenience while the others are computed from (2.2):

$$
\begin{aligned}
& \xi_{0}=\xi_{3}=\xi_{6}=(0,0,1) \\
& \xi_{1}=\left(\frac{4}{5}, 0, \frac{3}{5}\right) \\
& \xi_{2}=\left(-\frac{\sqrt{1-25 \epsilon^{2}}}{\sqrt{5\left(1-20 \epsilon^{2}\right)}}, \frac{5 \epsilon}{\sqrt{5\left(1-20 \epsilon^{2}\right)}},-\frac{2 \sqrt{1-25 \epsilon^{2}}}{\sqrt{5\left(1-20 \epsilon^{2}\right)}}\right) \\
& \xi_{4}=\left(-\frac{\sqrt{1-25 \epsilon^{2}}}{\sqrt{5\left(1-20 \epsilon^{2}\right)}}, \frac{5 \epsilon}{\sqrt{5\left(1-20 \epsilon^{2}\right)}}, \frac{2 \sqrt{1-25 \epsilon^{2}}}{\sqrt{5\left(1-20 \epsilon^{2}\right)}}\right) \\
& \xi_{5}=\left(\frac{4}{5}, 0,-\frac{3}{5}\right) .
\end{aligned}
$$




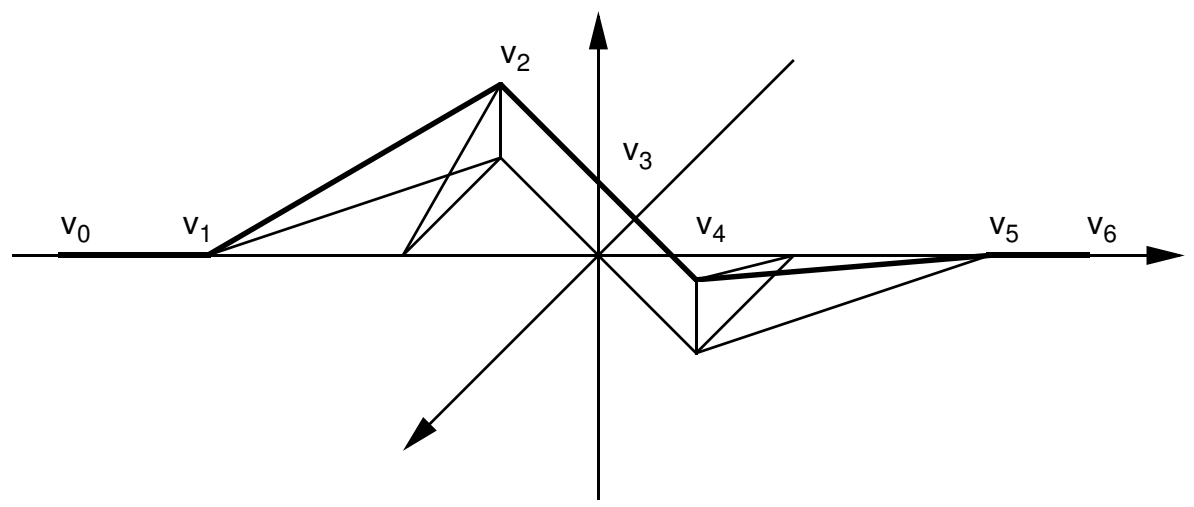

Figure 2.3

The change of base matrices are computed from (2.4) and (2.5):

$$
\begin{aligned}
& \Phi_{1}=-\Phi_{6}=\Psi^{*} \equiv\left[\begin{array}{cc}
\frac{3}{5} & \frac{4}{5} \\
-\frac{4}{5} & \frac{3}{5}
\end{array}\right], \\
& \Phi_{2}=\Phi_{3}=-\Phi_{4}=\Phi_{5}=\Psi_{\epsilon} \equiv\left[\begin{array}{cc}
-\frac{2 \sqrt{1-25 \epsilon^{2}}}{\sqrt{5\left(1-20 \epsilon^{2}\right)}} & \frac{1}{\sqrt{5\left(1-20 \epsilon^{2}\right)}} \\
-\frac{1}{\sqrt{5\left(1-20 \epsilon^{2}\right)}} & -\frac{2 \sqrt{1-25 \epsilon^{2}}}{\sqrt{5\left(1-20 \epsilon^{2}\right)}}
\end{array}\right] .
\end{aligned}
$$

Since the two negative signs in the matrix product will cancel, we have

$$
\Phi_{6} \circ \cdots \circ \Phi_{1}=\Psi^{*} \circ \Psi_{\epsilon}^{4} \circ \Psi^{*} .
$$

Therefore parallel translation from $T_{1}^{\perp}$ to $T_{6}^{\perp}$ is realized as rotation through the angle $2 \psi^{*}+4 \psi_{\epsilon}$. Observe that this angle increases monotonically from 0 to $\pi$ as $\epsilon$ goes from 0 to $\sqrt{7 / 180}$. Indeed, by (2.7), $\psi^{*}=\arcsin (-4 / 5)$, and, by (2.8), as $\epsilon$ goes from 0 to $\frac{1}{5}$, the angle $\psi_{\epsilon}$ increases from $-\arccos \left(-\frac{2}{\sqrt{5}}\right)$ to $-\pi / 2$, which is a change in angle exceeding $\pi / 4$. A simple calculation shows the angle change is equal to $\pi / 4$ when $\epsilon=\sqrt{7 / 180}$.

Lemma 2.5. Given any polygonal knot $K$, there is a family of polygonal knots $K_{\epsilon}, 0 \leq \epsilon \leq \sqrt{7 / 180}$, such that

(1) $K_{0}=K$, and

(2) $\mathrm{HOL}\left(K_{\epsilon}\right)$ continuously increases monotonically through an angle equal to $\pi$ as $\epsilon$ runs from 0 to $\sqrt{7 / 180}$.

Proof. Pick a unit vector $U$ which is perpendicular to the first edge of $K$. There is a unique orientation preserving similarity transformation $S$ of $\mathbf{R}^{3}$ into itself that carries the oriented line segment from $(0,-3,0)$ to $(0,3,0)$ onto the first edge of $K$ and the $z$-axis onto a positive multiple of $U$. Define $K_{\epsilon}$ to be $K$ with the first edge replaced by $S\left(E_{\epsilon}^{\delta}\right)$, where $E_{\epsilon}^{\delta}$ is as in 
Example 2.4, and where $\delta$ is chosen small enough so that $S\left(E_{\epsilon}^{\delta}\right)$ does not intersect any remaining edges of $K$, except for the endpoints of the first edge. This ensures that $K_{\epsilon}$ is a family of embeddings. Since the normal holonomy is unaffected under similarity transformations, the computation of Example 2.4 shows that $K_{\epsilon}$ has the desired properties.

Remark. Since reflection through a plane changes the sign of holonomy, the construction in Lemma 2.5 produces an isotopy $\bar{K}_{\epsilon}$ of $K$ such that the holonomy decreases monotonically through an angle of $-\pi$ as $\epsilon$ runs from 0 to $\sqrt{7 / 180}$ when an edge of $K$ is replaced with an appropriately scaled reflection of $E_{\epsilon}^{\delta}$.

\section{The writhing number of polygonal knots.}

The same double integral used to define the writhing number of a smooth knot is used to define the writhing number of a polygonal knot $K$.

$$
W R(K)=\frac{1}{4 \pi} \int_{0}^{2 \pi} \int_{0}^{2 \pi} \frac{K^{\prime}(s) \times K^{\prime}(t) \cdot(K(s)-K(t))}{|K(s)-K(t)|^{3}} \mathrm{~d} s \mathrm{~d} t .
$$

The integral is convergent and can be given by a simple formula involving the vertices. To see this, let $t_{0}<t_{1}<\cdots<t_{n}=t_{0}$ be the subdivision of $S^{1}$ into subintervals on which $K$ is linear. Set

$$
W_{i j}=\frac{1}{4 \pi} \int_{t_{j-1}}^{t_{j}} \int_{t_{i-1}}^{t_{i}} \frac{K^{\prime}(s) \times K^{\prime}(t) \cdot(K(s)-K(t))}{|K(s)-K(t)|^{3}} \mathrm{~d} s \mathrm{~d} t .
$$

When $i=j$, the vectors $K^{\prime}(s), K^{\prime}(t)$, and $(K(s)-K(t))$ are colinear, and when $|i-j|=1$, they are coplanar. Thus, if $|i-j| \leq 1, W_{i j}=0$, because the integrand is zero almost everywhere. Therefore,

$$
W R(K)=\sum_{|i-j| \geq 2} W_{i j}
$$

For a polygonal knot $K: S^{1} \rightarrow \mathbf{R}^{3}$, consider the Gauss mapping

$$
\gamma: S^{1} \times S^{1}-\Delta \rightarrow S^{2}
$$

defined by

$$
\gamma(s, t)=\frac{K(s)-K(t)}{|K(s)-K(t)|}
$$

where $\Delta$ is the diagonal of $S^{1} \times S^{1}$. When $|i-j| \geq 2$, the rectangle $\left[t_{i-1}, t_{i}\right] \times$ $\left[t_{j-1}, t_{j}\right]$ does not meet $\Delta$, and its image under $\gamma$ is a convex, geodesic quadrilateral $Q_{i j}$ in $S^{2}$, which is possibly degenerate since the $i$-th and $j$-th edges of $K$ could be coplanar. When non-degenerate, $\gamma$ carries $\left[t_{i-1}, t_{i}\right] \times$ $\left[t_{j-1}, t_{j}\right]$ diffeomorphically onto $Q_{i j}$. 
Lemma 3.1. Suppose $|i-j| \geq 2$, then

$$
4 \pi W_{i j}= \pm \operatorname{Area}\left(Q_{i j}\right)
$$

where Area $\left(Q_{i j}\right)$ is the area of $Q_{i j}$. The plus sign is taken when $\gamma$ is orientation reversing on $\left[t_{i-1}, t_{i}\right] \times\left[t_{j-1}, t_{j}\right]$.

Proof. Because

$$
4 \pi W_{i j}=-\int_{\left[t_{i-1}, t_{i}\right] \times\left[t_{j-1}, t_{j}\right]} \gamma^{*}\left(\omega_{S^{2}}\right),
$$

where $\omega_{S^{2}}$ is the area form on $S^{2}$ oriented by the outward normal, we have $4 \pi W_{i j}= \pm$ Area $\left(Q_{i j}\right)$. (See $[\mathbf{1 8}, \mathrm{p} .132]$.) The sign is positive if and only if $\gamma$ is orientation reversing on the rectangle $\left[t_{i-1}, t_{i}\right] \times\left[t_{j-1}, t_{j}\right]$. This occurs precisely when the scalar triple product $T_{i} \times T_{j} \cdot \gamma\left(t_{i}, t_{j}\right)>0$.

Suppose $|i-j| \geq 2$. Let $A=K\left(t_{j-1}\right), B=K\left(t_{j}\right), C=K\left(t_{i-1}\right)$, and $D=K\left(t_{i}\right)$. Let $\beta_{A D}, \beta_{A C}, \beta_{B D}$, and $\beta_{B C}$ denote the interior dihedral angles of the tetrahedron $A B C D$ at the respective edges $A D, A C, B D$, and $B C$. Each of these four dihedral angles corresponds under $\gamma$ to one of the four interior angles of $Q_{i j}$ having the same measure. Thus the area of $Q_{i j}$ is given by the excess formula:

$$
\operatorname{Area}\left(Q_{i j}\right)=\beta_{A D}+\beta_{A C}+\beta_{B D}+\beta_{B C}-2 \pi .
$$

Since the dihedral angles can be computed in terms of the vertices $A, B$, $C$, and $D$, this results in a formula for $W_{i j}$ in terms of the vertices (cf. [4, pp. 15-17] and [2, p. 1177]).

Proposition 3.2. For any polygonal knot $K$,

$$
2 \pi W R(K) \equiv \operatorname{HOL}(K) \bmod 2 \pi .
$$

Remark. As noted in the introduction, the same formula (1.2) holds for smooth knots.

Proof. If the vertices of $K$ are in general position, then $W R(K)+$ $(1 / 2 \pi) T T(K)$ is an integer by $[\mathbf{2}$, Theorem 4] where $T T(K)$ is the total torsion. Combining this with the previous remark, HOL $(K) \equiv-T T(K)$ $\bmod 2 \pi$, shows that (3.4) holds when the vertices of $K$ are in general position.

If the vertices of $K$ are not in general position, (3.4) still holds by continuity of $W R$ and HOL because $K$ can be approximated by polygonal knots whose vertices are in general position.

For sufficiently small $\epsilon$, the $\epsilon$-tube about a polygonal knot $K$ is a surface homeomorphic to the torus built out of pieces of cylinders which are pasted together along elliptical boundary curves. Each cylindrical piece of the tube is associated to an edge of $K$ which forms part of the axis of the cylinder. Moreover, each cylindrical piece is striated by a family of line segments which 
are parallel to the associated edge of $K$. There is an obvious correspondence between the set of stria and the set of directions in the plane perpendicular to the associated edge. Each stria in the $i$-th cylindrical piece connects to a unique stria in the adjoining $(i+1)$-st cylindrical piece in such a way that the corresponding directions $U_{i} \in T_{i}^{\perp}$ and $U_{i+1} \in T_{i+1}^{\perp}$ are related by $R_{i}\left(U_{i}\right)=U_{i+1}$ where $R_{i}$ is the rotation about the $i$-th vertex of $K$ described in $\S 2$. Therefore, if $K$ has zero holonomy modulo $2 \pi$, one of these stria, followed around the knot, will return to itself to produce a closed polygonal curve $J$ disjoint from $K$. Any two such $J$ are isotopic with one another. Thus, the linking number of $K$ with $J$ is independent of the choice of a sufficiently small $\epsilon$ and the choice of the stria. We define the autoparallel linking number of the polygonal knot $K$ with zero holonomy to be the linking number of $K$ with any such $J$.

Remark. The autoparallel linking number is different from the self-linking number defined in [2] for polygonal knots in general position.

Proposition 3.3. For polygonal knots $K$ of zero holonomy, $W R(K)$ is equal to the autoparallel linking number of $K$.

Proof. By rounding out the corners of $K$ we may replace $K$ by a smooth knot, still denoted $K$, with zero holonomy and the same writhe. Let $U$ be a smooth parallel normal vector field along $K$. Then as in $\S 1$, the twist of $U$ along $K$ is zero. Moreover, $L K(K, U)$ equals the autoparallel linking number of $K$. Therefore $W R(K)$ equals the autoparallel linking number by applying Călugăreanu's formula (1.1).

Remark. This shows that for oriented knots constructed from oriented knot diagrams as in Example 2.2, the writhing number is computed as the linking number of the oriented knot diagram and its blackboard framing, and therefore is given by the well-known combinatorial formula, see for example $[14$, p. 163].

\section{The space of polygonal knots.}

Given a finite subset $\sigma=\left\{t_{0}<t_{1}<\cdots<t_{n}=t_{0}\right\}$ of $S^{1}$, let $\mathcal{K}(\sigma)$ denote the collection of all polygonal knots $K: S^{1} \rightarrow \mathbf{R}^{3}$ with vertices $K\left(t_{i}\right), t_{i} \in \sigma$. Because a piecewise linear map $K: S^{1} \rightarrow \mathbf{R}^{3}$ is determined by its values at the vertices, $\mathcal{K}(\sigma)$ is in one-to-one correspondence with an open subset of $\mathbf{R}^{3 n}$. The correspondence takes a knot $K$ in $\mathcal{K}(\sigma)$ to the ordered $n$-tuple of vectors $\left(K\left(t_{1}\right), \ldots, K\left(t_{n}\right)\right)$. Topologize $\mathcal{K}(\sigma)$ so that the correspondence is a homeomorphism.

The family $\mathcal{F}$ of all finite subsets of $S^{1}$ is partially ordered by set inclusion. The collection of the $\mathcal{K}(\sigma)$ for $\sigma \in \mathcal{F}$ form a directed system of topological spaces. For all $\sigma_{1}, \sigma_{2}$ in $\mathcal{F}, \mathcal{K}\left(\sigma_{1}\right)$ is a closed subspace of $\mathcal{K}\left(\sigma_{2}\right)$ if and only 
if $\sigma_{1} \subset \sigma_{2}$, and always $\mathcal{K}\left(\sigma_{1}\right) \cap \mathcal{K}\left(\sigma_{2}\right)=\mathcal{K}\left(\sigma_{1} \cap \sigma_{2}\right)$ and $\mathcal{K}\left(\sigma_{1}\right) \cup \mathcal{K}\left(\sigma_{2}\right) \subset$ $\mathcal{K}\left(\sigma_{1} \cup \sigma_{2}\right)$.

Define the space of polygonal knots to be the union

$$
\mathcal{K}=\bigcup_{\sigma \in \mathcal{F}} \mathcal{K}(\sigma)
$$

topologized with the direct limit topology. This means that $\mathcal{K}$ has the weak topology, that is, a subset is closed in $\mathcal{K}$ if and only if its intersection with every $\mathcal{K}(\sigma)$ is closed. See [12, Chapter 15] or [21, pp. 27-28].

Lemma 4.1. $\mathcal{K}$ is Hausdorff.

Proof. Given two distinct polygonal knots $K_{1}$ and $K_{2}$ in $\mathcal{K}$, there exists a $t^{*} \in S^{1}$ such that $K_{1}\left(t^{*}\right) \neq K_{2}\left(t^{*}\right)$. But the evaluation map

$$
e v_{t^{*}}: \mathcal{K} \rightarrow \mathbf{R}^{3}
$$

defined by $e v_{t^{*}}(K)=K\left(t^{*}\right)$ is continuous on $\mathcal{K}(\sigma)$ for every $\sigma \in \mathcal{F}$. Therefore $e v_{t^{*}}$ is continuous on $\mathcal{K}$, since $\mathcal{K}$ has the limit topology. If $U_{1}$ and $U_{2}$ are disjoint open sets in $\mathbf{R}^{3}$ containing respectively $K_{1}\left(t^{*}\right)$ and $K_{2}\left(t^{*}\right)$, then $e v_{t^{*}}^{-1}\left(U_{1}\right)$ and $e v_{t^{*}}^{-1}\left(U_{2}\right)$ are disjoint open sets in $\mathcal{K}$ containing respectively $K_{1}$ and $K_{2}$.

By applying Lemma 15.10 in [12] we have the following result.

Lemma 4.2. Every compact subset of $\mathcal{K}$ is contained in some $\mathcal{K}(\sigma)$.

Remark. In view of Lemma 4.2, every path in $\mathcal{K}$ is a path in some $\mathcal{K}(\sigma)$. Thus paths in $\mathcal{K}$ correspond to isotopies of polygonal knots, and vice versa.

\section{Serre fibrations over circles.}

Recall that a continuous map $f: X \rightarrow B$ is a Serre fibration if $f$ has the homotopy lifting property with respect to simplices $\Delta^{n}$ for all $n$. (See [12, p. 79] and [21, p. 29].) There is a long exact homotopy sequence associated to a Serre fibration [12, p. 84]. This section is devoted to proving a characterization of Serre fibrations over the circle.

Proposition 5.1. Let $f: X \rightarrow S^{1}$ be a continuous map from a topological space $X$ to the circle. Then $f$ is a Serre fibration if and only if for every continuous simplex $s: \Delta^{n} \rightarrow X$ there exists a pair of homotopies

$$
h, \bar{h}: \Delta^{n} \times[0,1] \rightarrow X
$$

such that, for every $x \in \Delta^{n}$,

(1) $h(x, 0)=\bar{h}(x, 0)=s(x)$,

(2) $f(h(x, t))$ continuously increases monotonically through an angle equal to $\pi$ as $t$ runs from 0 to 1 , and 
(3) $f(\bar{h}(x, t))$ continuously decreases monotonically through an angle equal to $-\pi$ as $t$ runs from 0 to 1 .

The proof is based on several lemmas.

The exponential map exp : $\mathbf{R} \rightarrow S^{1}$ is a covering map. Consider the pullback $\widetilde{X}$ of this covering space by the continuous map $f: X \rightarrow S^{1}$. Then there is a covering map EXP: $\widetilde{X} \rightarrow X$ and a bundle map $\widetilde{f}: \widetilde{X} \rightarrow \mathbf{R}$ such that the following diagram commutes.

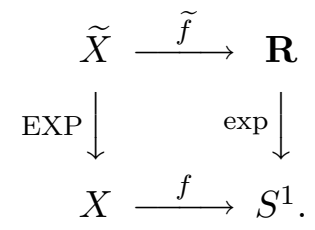

The following lemma is obvious (cf. [21, pp. 36-37]).

Lemma 5.2. $f: X \rightarrow S^{1}$ is a Serre fibration if and only if $\widetilde{f}: \widetilde{X} \rightarrow \mathbf{R}$ is a Serre fibration.

Lemma 5.3. The map $f: X \rightarrow S^{1}$ satisfies the hypothesis of Proposition 5.1. if and only if for every positive integer $m$ and every continuous simplex $s: \Delta^{n} \rightarrow \widetilde{X}$, there exists a homotopy $h_{m}: \Delta^{n} \times[-m, m] \rightarrow \widetilde{X}$ such that, for every $x \in \Delta^{n}$,

(1) $h_{m}(x, 0)=s(x)$,

(2) $t \mapsto \tilde{f}\left(h_{m}(x, t)\right)$ is monotone increasing, and

(3) $\widetilde{f}\left(h_{m}(x, m)\right)-\widetilde{f}\left(h_{m}(x, 0)\right) \geq m \pi$ and $\widetilde{f}\left(h_{m}(x, 0)\right)-\widetilde{f}\left(h_{m}(x,-m)\right) \geq$ $m \pi$.

Proof. Assuming $f$ satisfies the hypothesis of Proposition 5.1, this is proved by induction on $m$. To construct $h_{1}$, patch together the lifts from $X$ to $\widetilde{X}$ of the homotopies $h$ and $\bar{h}$ obtained from the simplex EXPos: $\Delta^{n} \rightarrow X$, which exist according to the hypothesis of Proposition 5.1. Then

$$
\operatorname{EXP}\left(h_{1}(x, t)\right)=\left\{\begin{array}{llr}
\bar{h}(x,-t) & \text { if } & -1 \leq t \leq 0 \\
h(x, t) & \text { if } & 0 \leq t \leq 1 .
\end{array}\right.
$$

Once $h_{m}$ has been constructed, let $\bar{h}$ be the homotopy in $X$ obtained from the simplex $x \mapsto \operatorname{EXP}\left(h_{m}(x,-m)\right)$ by hypothesis, and let $h$ be the homotopy obtained from the simplex $x \mapsto \operatorname{EXP}\left(h_{m}(x, m)\right)$. Then $h_{m+1}$ is constructed as an extension of $h_{m}$ by patching the homotopy $\bar{h}$ on the left of $h_{m}$ and patching the homotopy $h$ on the right of $h_{m}$ after suitable reparametrizations of the interval domains of $\bar{h}$ and $h$.

The converse is obvious.

Lemma 5.4. A continuous map $\widetilde{f}: \widetilde{X} \rightarrow \mathbf{R}$ is a Serre fibration if and only if for every positive integer $m$ and every continuous simplex $s: \Delta^{n} \rightarrow \widetilde{X}$ there exists a homotopy $h_{m}: \Delta^{n} \times[-m, m] \rightarrow \widetilde{X}$ such that, for every $x \in \Delta^{n}$, 
(1) $h_{m}(x, 0)=s(x)$,

(2) $t \mapsto \widetilde{f}\left(h_{m}(x, t)\right)$ is monotone increasing, and

(3) $\tilde{f}\left(h_{m}(x, m)\right)-\widetilde{f}\left(h_{m}(x, 0)\right) \geq m \pi$ and $\widetilde{f}\left(h_{m}(x, 0)\right)-\widetilde{f}\left(h_{m}(x,-m)\right) \geq$ $m \pi$.

Proof. Let $s: \Delta^{n} \rightarrow \widetilde{X}$ be a continuous simplex in $\widetilde{X}$, and let $k: \Delta^{n} \times$ $[0,1] \rightarrow \mathbf{R}$ be a homotopy of $\widetilde{f} \circ s$. Thus we have the following commutative diagram where $i_{0}(x)=(x, 0)$ for all $x$ in $\Delta^{n}$.

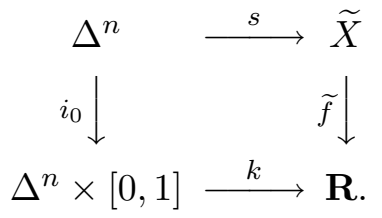

Pick an integer $m>0$ so that

$$
|k(x, t)-k(x, 0)|<m \pi
$$

for all $x$ in $\Delta^{n}$ and $t$ in $[0,1]$. Consider the map

$$
\left(i d, \tilde{f} \circ h_{m}\right): \Delta^{n} \times[-m, m] \rightarrow \Delta^{n} \times \mathbf{R} .
$$

By (2), this is one-to-one, and hence a homeomorphism onto its image. Consider also the map

$$
(i d, k): \Delta^{n} \times[0,1] \rightarrow \Delta^{n} \times \mathbf{R} .
$$

By (3), the image of the map $(i d, k)$ is contained in the image of the map $\left(i d, \tilde{f} \circ h_{m}\right)$.

It is straightforward to verify that the map

$$
h_{m} \circ\left(i d, \tilde{f} \circ h_{m}\right)^{-1} \circ(i d, k): \Delta^{n} \times[0,1] \rightarrow \widetilde{X}
$$

is a lift of $k$. Here are the details of the calculation:

$$
\begin{aligned}
& h_{m} \circ\left(i d, \tilde{f} \circ h_{m}\right)^{-1} \circ(i d, k)(x, 0) \\
& =h_{m} \circ\left(i d, \tilde{f} \circ h_{m}\right)^{-1}(x, k(x, 0)) \\
& =h_{m} \circ\left(i d, \tilde{f} \circ h_{m}\right)^{-1}(x, \widetilde{f}(s(x))) \\
& =h_{m} \circ\left(i d, \tilde{f} \circ h_{m}\right)^{-1}\left(x, \tilde{f}\left(h_{m}(x, 0)\right)\right) \\
& =h_{m}(x, 0)=s(x)
\end{aligned}
$$

and

$$
\begin{aligned}
& \tilde{f} \circ h_{m} \circ\left(i d, \tilde{f} \circ h_{m}\right)^{-1} \circ(i d, k)(x, t) \\
& =\tilde{f} \circ h_{m} \circ\left(i d, \tilde{f} \circ h_{m}\right)^{-1}(x, k(x, t)) \\
& =\tilde{f} \circ h_{m}\left(x, t^{\prime}\right) \text { where } \tilde{f}\left(h_{m}\left(x, t^{\prime}\right)\right)=k(x, t) \\
& =k(x, t) .
\end{aligned}
$$


This proves that $\tilde{f}$ is a Serre fibration.

Conversely, suppose $\widetilde{f}$ is a Serre fibration. Given a positive integer $m$ and a continuous simplex $s: \Delta^{n} \rightarrow \widetilde{X}, h_{m}$ can be constructed from the homotopy lifting property by lifting the homotopy $k: \Delta^{n} \times[-m, m] \rightarrow \mathbf{R}$ defined by the formula $k(x, t)=\widetilde{f}(s(x))+t \pi$ to $\widetilde{X}$.

\section{HOL and WR are Serre fibrations.}

The formula in $\S 2$ shows that the normal holonomy of a polygonal knot depends continuously on the vertices. Thus HOL: $\mathcal{K} \rightarrow S^{1}$ is continuous because $\mathcal{K}$ has the limit topology.

Theorem 6.1. HOL $: \mathcal{K} \rightarrow S^{1}$ is a Serre fibration.

Proof. Let $s: \Delta^{n} \rightarrow \mathcal{K}$ be a continuous simplex in $\mathcal{K}$. Since $\Delta^{n}$ is compact, Lemma 4.2 implies there exists a finite set $\sigma \subset S^{1}$ such that $s\left(\Delta^{n}\right) \subset \mathcal{K}(\sigma)$. Moreover, there exists a continuous unit vector field $U: \Delta^{n} \rightarrow \mathbf{R}^{3}$ such that $U(x)$ is perpendicular to the first edge of the knot $s(x)$ for every $x$ in $\Delta^{n}$. To prove this, consider the vector bundle over $\Delta^{n}$ such that the fiber over $x \in \Delta^{n}$ is the vector subspace of $\mathbf{R}^{3}$ consisting of all vectors perpendicular to the first edge of the knot $s(x)$. This bundle must be trivial because the base space $\Delta^{n}$ is contractible [13, p. 29]. Hence this bundle admits a continuous nonzero section, which after normalizing gives the unit vector field $U$.

For each $x \in \Delta^{n}$, consider the construction in Lemma 2.5 of the family of knots starting from $K=s(x)$ with $U=U(x)$. For any fixed $\delta$, the construction depends continuously on $K$ and the choice of $U$. Because $\Delta^{n}$ is compact, there is a value of $\delta$ that can be used uniformly for all $x \in \Delta^{n}$. Changing the parameter interval to $[0,1]$ produces a homotopy $h: \Delta^{n} \times$ $[0,1] \rightarrow \mathcal{K}$ such that, for all $x \in \Delta^{n}, h(x, 0)=s(x)$ and $t \mapsto \operatorname{HOL}(h(x, t))$ is strictly monotone increasing through an angle equal to $\pi$ as $t$ runs from 0 to 1. Likewise, in view of the remark after Lemma 4.2, there is also a homotopy $\bar{h}: \Delta^{n} \times[0,1] \rightarrow \mathcal{K}$ which decreases holonomy monotonically through an angle $-\pi$. Proposition 5.1 implies that HOL is a Serre fibration.

Theorem 6.2. $W R: \mathcal{K} \rightarrow \mathbf{R}$ is a Serre fibration.

Proof. By Proposition 3.2, HOL factors as the composition of the map $2 \pi W R: \mathcal{K} \rightarrow \mathbf{R}$ and the covering map exp $: \mathbf{R} \rightarrow S^{1}$. Thus, because HOL is a Serre fibration, so will be the map $2 \pi W R: \mathcal{K} \rightarrow \mathbf{R}$. Therefore WR is a Serre fibration.

Corollary 6.3. Two isotopic polygonal knots which have the same writhing number are isotopic through a family of polygonal knots with the same writhing number.

Proof. For each real number $w$, the long exact homotopy sequence for a fibration, together with the fact that $\mathbf{R}$ is contractible, implies that the 
inclusion of the fiber $W R^{-1}(w)$ into $\mathcal{K}$ is a weak homotopy equivalence. In particular,

$$
W R_{*}: \pi_{0}\left(W R^{-1}(w)\right) \rightarrow \pi_{0}(\mathcal{K})
$$

is an isomorphism of sets. Thus the path connected components of $W R^{-1}(w)$ are in one-to-one correspondence with the path connected components of $\mathcal{K}$.

Corollary 6.4. A polygonal knot with zero writhe is isotopic to its reflection if and only if it is isotopic to its reflection through polygonal knots with zero writhe.

Proof. This follows from Corollary 6.3 because the writhe of the reflection of a polygonal knot is the negative of the writhe of the knot.

Remark. This is a three dimensional version of the Mirror Theorem $[\mathbf{1 4}$, p. 173].

Reversing the orientation of a polygonal knot does not change the writhing number. Therefore we have the following:

Corollary 6.5. A polygonal knot is isotopic to its inverse if and only if it is isotopic to its inverse through a family of polygonal knots of the same writhe.

The class of a polygonal knots of zero holonomy will be denoted $\mathcal{K}_{0}$. This class is a fiber of HOL . Consider part of the long exact homotopy sequence for $\mathrm{HOL}$ :

$$
\rightarrow \pi_{1}(\mathcal{K}) \stackrel{\mathrm{HOL}^{*}}{\rightarrow} \pi_{1}\left(S^{1}\right) \stackrel{\partial}{\rightarrow} \pi_{0}\left(\mathcal{K}_{0}\right) \rightarrow \pi_{0}(\mathcal{K}) .
$$

By Proposition 3.2, HOL factors through $\mathbf{R}$. Thus $\mathrm{HOL}_{*}$ is the trivial group homomorphism, and the above long exact sequence gives the short exact sequence

$$
1 \rightarrow \pi_{1}\left(S^{1}\right) \stackrel{\partial}{\rightarrow} \pi_{0}\left(\mathcal{K}_{0}\right) \rightarrow \pi_{0}(\mathcal{K}) .
$$

This shows that the path connected components of $\mathcal{K}_{0}$ are indexed by the set of integers in the form of $\pi_{1}\left(S^{1}\right)$ and the path connected components of $\mathcal{K}$. By Corollary 6.3, WR is constant on each path connected component of $\mathcal{K}_{0}$. By Proposition 3.3, this constant is an integer equal to the autoparallel linking number of any knot in that component. Therefore the following holds:

Corollary 6.6. Two isotopic knots having zero holonomy are isotopic through a family of such knots if and only if they have the same autoparallel linking number.

Recall that the fundamental group of the base of a Serre fibration acts on the set of path connected components of the fiber (cf. [21, p. 186]). Thus, by Theorem $6.2, \pi_{1}\left(S^{1}\right) \approx \mathbf{Z}$ acts on the set $\pi_{0}\left(\mathcal{K}_{0}\right)$. Let $n \cdot[K]$ denote the action of $n \in \mathbf{Z}$ on $[K]$ in $\pi_{0}\left(\mathcal{K}_{0}\right)$. In view of Corollary 6.5 , we can define writhe on $\pi_{0}\left(\mathcal{K}_{0}\right)$ by $W R([K])=W R(K)$. 
Theorem 6.7. For every $[K]$ in $\pi_{0}\left(\mathcal{K}_{0}\right)$ and $n$ in $\mathbf{Z}$,

$$
W R(n \cdot[K])=W R([K])+n .
$$

If $K$ is a knot diagram, then $1 \cdot[K]$ has a knot diagram representative that results from $K$ by a type I Reidemeister move.

Proof. Let $[K] \in \pi_{0}\left(\mathcal{K}_{0}\right)$ and $n \in \mathbf{Z}$. Consider the commutative diagram:

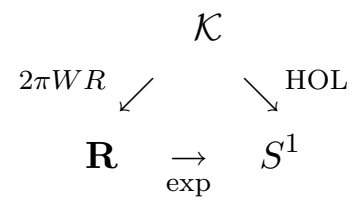

The loop in $S^{1}$ representing $n$ lifts to a curve $\alpha$ in $\mathcal{K}$ starting at $K$ and ending in $n \cdot[K]$. This lift can be achieved in two stages. First the loop lifts to a path in $\mathbf{R}$ starting at $2 \pi W R(K)$ and ending at $2 \pi(W R(K)+n)$, which then lifts to the curve $\alpha$. This proves the first statement.

If $K$ is a knot diagram, it defines a knot class $[K]$ in $\pi_{0}\left(\mathcal{K}_{0}\right)$ by Example 2.2. Rearrange the diagram $K$ to $K_{0}$ as shown in Figure 6.1. By rotating the triangle $A B C$ around the line $A C$, one produces a family of knots $K_{\theta}, 0 \leq \theta \leq \pi$, such that $\operatorname{HOL}\left(K_{\theta}\right)=2 \theta$ just as in Example 2.3. Since $\operatorname{HOL}\left(K_{\theta}\right)$ wraps once counterclockwise around the circle, $\left[K_{\pi}\right]=(+1) \cdot[K]$. Clearly, the diagram for $K_{\pi}$ results from that of $K$ by a type I Reidemeister move. See Figure 6.1. (For a description of the three types of Reidemeister moves see $[\mathbf{1 4}$, p. 9].)

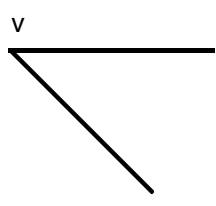

$\mathrm{K}$
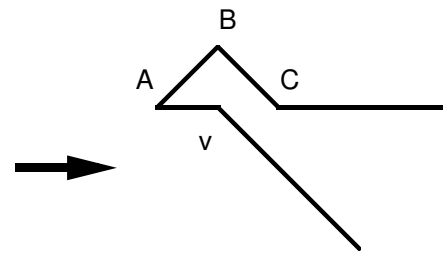

$\mathrm{K}_{0}$

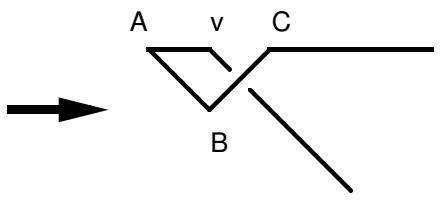

$\mathrm{K}_{\pi}$

Figure 6.1

\section{Immersed polygonal knots.}

A piecewise linear immersion of $S^{1}$ into $\mathbf{R}^{3}$ is a piecewise linear map which is locally an embedding. We will call such maps immersed polygonal knots. The notation in $\S 2$ carries over to immersed polygonal knots. It is still the case that the exterior angle at every vertex must be strictly less than $\pi$, even though an immersed polygonal knot may have self-intersections. Thus normal holonomy is defined for immersed polygonal knots. However, the 
writhing number cannot be defined, and there is no analogue for Proposition 3.2 .

On the other hand, there is an analogue for the autoparallel linking number of a polygonal knot of zero holonomy. Let $K$ be an immersed polygonal knot of zero holonomy. Recalling the notation of $\S 2$, pick a unit vector $U_{1} \in T_{1}^{\perp}$, and define $U_{i} \in T_{i}^{\perp}$ inductively by $U_{i+1}=R_{i}\left(U_{i}\right)$. Since $K$ has zero holonomy, $R_{n}\left(U_{n}\right)=U_{1}$ by (2.3). Let $M_{i}$ be the orthogonal matrix in $S O(3)$ whose columns are the vectors $T_{i}, U_{i}, T_{i} \times U_{i}$. Recalling Formula $(2.2)$, define $\Gamma_{i}^{\theta}$ to be rotation about $\xi_{i}$ through an angle $\theta$, where $0 \leq \theta \leq \alpha_{i}$. Then $\theta \mapsto \Gamma_{i}^{\theta} \circ M_{i}$ is a path in $S O(3)$ joining $M_{i}$ to $M_{i+1}$. The collection of these paths forms a loop in $S O(3)$ whose free homotopy class is independent of the choice of $U_{1}$. The free homotopy class of loops in $S O(3)$ associated to $K$ in this way will be called the rotation class of $K$. There are two possible rotation classes because $\pi_{1}(S O(3)) \approx \mathbf{Z}_{2}$ (see, for example, [21, pp. 198-199]). Both rotation classes are realizable. For example, the rotation class of any regular planar polygon is the nontrivial homotopy class in $S O(3)$, while the rotation class of a polygonal planar figure eight is the trivial homotopy class.

The space $\mathcal{I}$ of immersed polygonal knots can be topologized as the direct limit of finite dimensional approximations $\mathcal{I}(\sigma)$ just as in $\S 4$. The proof of Theorem 6.1 goes over without modification. In fact it is a little easier since one need not worry about self-intersections introduced by the homotopies.

Theorem 7.1. HOL $: \mathcal{I} \rightarrow S^{1}$ is a Serre fibration.

Let $\mathcal{I}_{0}$ denote the space of immersed polygonal knots of zero holonomy. Consider the long exact homotopy sequence of HOL :

$$
\rightarrow \pi_{1}(\mathcal{I}) \stackrel{\text { HOL }}{\rightarrow} \pi_{1}\left(S^{1}\right) \stackrel{\partial}{\rightarrow} \pi_{0}\left(\mathcal{I}_{0}\right) \rightarrow \pi_{0}(\mathcal{I}) .
$$

$\pi_{0}(\mathcal{I})$ is a set with one element since $\mathcal{I}$ is path connected. The loop $K_{\theta}$, $0 \leq \theta \leq 2 \pi$, of Example 2.3 shows that the image of $\mathrm{HOL}_{*}$ in $\mathbf{Z} \approx \pi_{1}\left(S^{1}\right)$ contains $2 \mathbf{Z}$. Thus $\pi_{0}\left(\mathcal{I}_{0}\right)$ has two elements corresponding to the two rotation classes. This proves the following analogue to Corollary 6.3. There is a similar result for smooth space curves of non-vanishing curvature [7].

Corollary 7.2. A pair of immersed polygonal knots of zero holonomy are isotopic through a family of immersed polygonal knots of zero holonomy if and only if they have the same rotation class.

Since $\mathcal{K}_{0} \subset \mathcal{I}_{0}$, every $K \in \mathcal{K}_{0}$ has a rotation class $R O T(K) \in \mathbf{Z}_{2} \approx$ $\pi_{1}(S O(3))$ as well as an autoparallel linking number, which equals $W R(K) \in$ $\mathbf{Z}$ by Proposition 3.3.

Corollary 7.3. Let $K$ be a polygonal knot of zero holonomy. Then

$$
W R(K)+1 \equiv R O T(K) \bmod 2 .
$$


Remark. This result should be compared with Fuller's formula [9, Equation 6.1] or [1, Equation 16].

Proof. If $K$ is a knot diagram, $\operatorname{ROT}(K)$ is clearly equal to the rotation number $[\mathbf{2 2}]$ of the diagram modulo 2. (See $[\mathbf{1 4}$, p. 170] for a combinatorial definition of rotation number.) Furthermore, the equivalence

$$
W R(K)+1 \equiv \operatorname{ROT}(K) \bmod 2
$$

holds for knot diagrams $K$ because it holds for the trivial knot diagram and the equivalence is unchanged under Reidermeister moves and crossing changes. Now observe every connected component of $\mathcal{K}_{0}$ contains a knot diagram as in Example 2.2 and Theorem 6.7. Because HOL and ROT are constant on the connected components of $\mathcal{K}_{0}$ by Corollaries 6.6 and 7.2, the equivalence must hold for every $K \in \mathcal{K}_{0}$.

\section{References}

[1] J. Aldinger, I. Klapper and M. Tabor, Formulae for the calculation and estimation of writhe, J. Knot Theory Ramifications, 4 (1994), 343-372, MR 97d:57003, Zbl 0840.57003.

[2] T. Banchoff, Self linking numbers of space polygons, Indiana U. Math. J., 12 (1976), 1171-1188, MR 55 \#4227, Zbl 0363.53002.

[3] T. Banchoff and J. White, The behavior of the total twist and self-linking number of a closed space curve under inversions, Math. Scand., 36 (1975), 254-262, MR 52 \#4312, Zbl 0326.53067.

[4] G. Călugăreanu, L'intégrale de Gauss et l'analyse des nœuds tridimensionnels, Rev. Math. Pures Appl., 4 (1959), 5-20, MR 24 \#A1693.

[5] __ Sur les classes d'isotopie des noeuds tridimensionnels et leurs invariants, Czechoslovak Math. J., 11 (1961), 588-625, MR 26 \#6868, Zbl 0118.16005.

[6] J. Cantarella, D. DeTurck and H. Gluck, Upper bounds for the writhing of knots and the Helicity of vector fields, preprint.

[7] E. Feldman, Deformations of closed space curves, J. Differential Geometry, 2 (1968), 67-75, MR 38 \#725, Zbl 0195.53401.

[8] F.B. Fuller, The writhing number of a space curve, Proc. Nat. Acad. Sci. USA, 68 (1971), 815-819, MR 43 \#3928, Zbl 0212.26301.

[9] _ Decomposition of the linking number of a closed ribbon: A problem from molecular biology, Proc. Nat. Acad. Sci. USA, 75 (1978), 3557-3561, MR 58 \#9367, Zbl 0395.92010.

[10] H. Gluck and L.-H. Pan, Knot theory in the presence of curvature, I, preprint.

[11] _ Embedding and knotting of positive curvature surfaces in 3-space, Topology, 37 (1998), 851-873, MR 98m:57008.

[12] B. Gray, Homotopy Theory: An Introduction to Algebraic Topology, Academic Press, New York, 1975, MR 53 \#6528, Zbl 0322.55001.

[13] D. Husemoller, Fibre Bundles, Springer Verlag, New York, 1975, MR 51 \#6805, Zbl 0307.55015. 
[14] L. Kauffman, On Knots, Annals of Math Studies, 115 (1987), Princeton University Press, Princeton, MR 89c:57005, Zbl 0627.57002.

[15] M. Levi, A "bicycle wheel" proof of the Gauss-Bonnet Theorem, Expositiones Mathematicae, 12 (1994), 145-164, MR 95j:53007, Zbl 0815.53002.

[16] D. Miller and C. Benham, Fixed-writhe isotopies and the topological conservation law for closed, circular DNA, J. Knot Theory Ramifications, 5 (1996), 859-866, MR 98b:57019, Zbl 0872.57013.

[17] H. Moffatt and R. Ricca, Helicity and the Călugăreanu invariant, Proc. Roy. Soc. London Ser. A, 439 (1992), 411-429, MR 94b:53006, Zbl 0771.57013.

[18] M. Spivak, Calculus on Manifolds, W.A. Benjamin, Inc., Menlo Park, 1965, MR 35 \#309, Zbl 0141.05403.

[19] M. Tabor and I. Klapper, Dynamics of twist and writhe and the modeling of bacterial fibers, in 'Mathematical approaches to biomolecular structure and dynamics (Minneapolis, MN, 1994),' IMA Vol. Math. Appl., 82, Springer, New York, (1996), 139-159, MR 97m:53004, Zbl 0864.92006.

[20] J. White, Twist invariants and the Pontryagin numbers of immersed manifolds, in 'Differential Geometry (Proc. Sympos. Pure Math., XXVII, Part 1)', American Mathematical Society, Providence, (1975), 429-437, MR 53 \#9248, Zbl 0336.57021.

[21] G. Whitehead, Elements of Homotopy Theory, Springer-Verlag, New York, 1978, MR 80b:55001, Zbl 0406.55001.

[22] H. Whitney, On regular closed curves in the plane, Compositio Math., 4 (1937), 276-284, Zbl 0016.13804.

Received July 11, 2000 and revised June 13, 2001.

Department of Mathematics

SAINT Louis UNIVERSITY

ST. Louis, MO 63103

E-mail address: hebdajj@slu.edu

Department of Mathematics

SAint Louis University

St. Louis, MO 63103

E-mail address: tsaumc@slu.edu 